\title{
Prevalence study of nosocomial infections in a north-Bari region hospital in the years 2007-20 I 0
}

Tito Del Gaudio', Maria Antonietta Distasi', Antonella De Toma², Rosaria Pandolfo², Giovanni Grilli²

I Laboratorio Analisi P.O. "Lorenzo Bonomo"Andria ASL BT

2 Direzione Sanitaria P.O. “Lorenzo Bonomo”Andria ASL BT

Key words: Nosocomial infections, prevalence study

Studio di prevalenza delle infezioni ospedaliere in un ospedale del nord-barese nel periodo 2007 2010

\section{SUMMARY}

Nosocomial infections, arising from complications during hospital treatment, represent one of the most important cause of disease. The Andria hospital has initiated a prevalence study, as a part of a general program promoted by the "Agenzia Regionale per la Sanità" (Regional Health Board) of Regione Puglia.

Samples were collected from patients hospitalized in the period 2007 - 2010. Risk factors, ongoing infections, selected antibiotic therapy, site of infection, microorganism types, as well as patient's clinic and personal data, were all elements taken into account.

The study shows that nosocomial infections, due to different health care related risk factors, are most frequently encountered in Intensive Care Units.

A poor adhesion to the Regione Puglia antibiotic prophylaxis guidelines was observed in surgery. An insufficient use of cultural methods to confirm/diagnose the presence of bacterial infections was also observed; this fact could lead to underestimate the incidence of nosocomial infections.

\section{INTRODUZIONE}

Le infezioni ospedaliere, meglio definite come infezioni correlate all'assistenza sanitaria, rappresentano una delle principali cause di malattia derivanti da complicanze nell'attività assistenziale.

Vengono definite infezioni ospedaliere quelle infezioni insorte durante la degenza ospedaliera che non erano presenti né in incubazione al momento del ricovero.

Nell'Ospedale di Andria è stato avviato uno studio di prevalenza nell'ambito di un programma promosso dall'ARES della Regione Puglia.

Lo studio si prefigge lo scopo di quantificare le infezioni nosocomiali, nonché di individuare quelle attività sanitarie che possono contribuire ad aumentare il rischio di infezioni contraibili in ospedale.

\section{MATERIALI E METODI}

Nel periodo di osservazione (2007-2010) sono stati arruolati tutti i pazienti ricoverati nella terza settimana di ottobre da non meno di $48 \mathrm{~h}$, escludendo quelli in dimissione.

I dati considerati, oltre a quelli anagrafici e clinici, comprendevano i fattori rischio, le even- tuali infezioni in atto, la terapia antibiotica instaurata, la sede di infezione ed i microrganismi isolati (5).

\section{RISULTATI}

Durante lo studio sono stati arruolati, su un totale di 528 degenti, 345 pazienti $(65.3 \%)$.

Di questi ultimi, 117 (33.9\%) erano portatori di catetere vescicale e $56(16.2 \%)$ di catetere venoso. $24(6.9 \%)$ pazienti erano sottoposti a ventilazione assistita e 70 (20.2\%) presentavano una ferita chirurgica (Tabella 1).

I pazienti sottoposti ad antibiotico-terapia erano in totale $216(62.6 \%)$.

99/216 (45.8\%) a scopo di profilassi, 99/216 per terapia empirica e $18(8.3 \%)$ per terapia sulla base dell'antibiogramma.

L'adesione alle linee guida regionali per la profilassi antibiotica in chirurgia (1) è risultata del $34.3 \%$ (Tabella 2).

Le infezioni batteriche riscontrate sono state $114(33.0 \%)$ di cui $31(8.9 \%)$ di eziologia nosocomiale. Gli esami colturali eseguiti sono stati 38 , il $33.3 \%$ del totale delle infezioni riscontrate.

\section{Corresponding author: Tito Del Gaudio}

Via Raffaello Sanzio, 16 - 7003। Andria (BT)

Tel.: 0883-2992 I 2 / 274 - FAX: 0883-299II8

E-mail: titodelgaudio@tiscali.it 
Undici casi (35.5\%) di infezione nosocomiale si sono verificati in reparti di area critica (rianimazione), 11 (35.5\%) in reparti dell'area medica e 9 $(29.0 \%)$ in quelli dell'area chirurgica (Tabella 3$)$.

\section{CONCLUSIONI}

Il nostro studio evidenzia, come rilevato da altri autori (2), che il reparto di Rianimazione, in rapporto al basso numero dei ricoveri annui, è quello nel quale più facilmente si possono verificare infezioni ospedaliere a causa dei molteplici fattori di rischio che concorrono nell'assistenza sanitaria.

Inoltre, in accordo con altre osservazioni (6), in questo reparto 5 casi di infezione delle basse vie respiratorie, per i quali è stato possibile individuare un'etiologia batterica, erano riconducibili a ventilazione forzata come principale fattore di rischio.

All'interno di vari reparti, 6/10 batteriemie erano riconducibili a cateterismo venoso, mentre tutte le infezioni a livello di cute e/o del connettivo potevano essere ricondotte alla presenza di ferite chirurgiche quale fattore di rischio.

Abbiamo rilevato, in contrasto con i dati della letteratura $(3,4)$, uno scarso numero di infezioni delle vie urinarie rispetto a quello dei pazienti cateterizzati.

Inoltre, nei primi 3 anni dello studio, abbiamo riscontrato una scarsa adesione alle linee guida dettate dall'A.Re.S. della Regione Puglia per la profilassi antibiotica in chirurgia, adesione nettamente migliorata nel 2010 dopo intervento della Direzione sanitaria e del CIO di Presidio.

Da segnalare, infine, l'insufficiente ricorso agli esami colturali per la diagnosi di infezione, condizione che potrebbe portare ad una sottostima delle infezioni ospedaliere.

Riteniamo quindi che lo Studio di Prevalenza possa essere un valido ausilio per conoscere la propria realtà riguardo le infezioni nosocomiali e per intervenire sui fattori di rischio laddove possibile.

Tabella I. Fattori di rischio

\begin{tabular}{|l|c|c|c|c|c|}
\hline & $\mathbf{2 0 0 7}$ & $\mathbf{2 0 0 8}$ & $\mathbf{2 0 0 9}$ & $\mathbf{2 0 1 0}$ & TOTALE \\
\hline N. PAZIENTI/ANNO & $\mathbf{8 3}$ & $\mathbf{9 1}$ & $\mathbf{9 3}$ & $\mathbf{7 8}$ & $\mathbf{3 4 5}$ \\
\hline Fattori di rischio & & & & & \\
\hline Catetere vescicale & $29(34.9 \%)$ & $30(33.0 \%)$ & $27(29.0 \%)$ & $31(39.7 \%)$ & $117(33.9 \%)$ \\
\hline Catetere venoso & $11(13.3 \%)$ & $5(5.5 \%)$ & $21(22.6 \%)$ & $19(24.4 \%)$ & $56(16.2 \%)$ \\
\hline Ventilazione assistita & $6(7.2 \%)$ & $5(5.5 \%)$ & $6(6.5 \%)$ & $7(9.0 \%)$ & $24(7.0 \%)$ \\
\hline Ferita chirurgica & $17(20.5 \%)$ & $19(20.9 \%)$ & $18(19.4 \%)$ & $16(20.5 \%)$ & $70(20.3 \%)$ \\
\hline
\end{tabular}

Tabella 2. Terapia antibiotica

\begin{tabular}{|l|c|c|c|c|c|}
\hline & $\mathbf{2 0 0 7}$ & $\mathbf{2 0 0 8}$ & $\mathbf{2 0 0 9}$ & $\mathbf{2 0 1 0}$ & TOTALE \\
\hline Terapia antibiotica/anno & $62(74.7 \%)$ & $45(49.5 \%)$ & $47(50.5 \%)$ & $62(79.5 \%)$ & $216(62.6 \%)$ \\
\hline profilassi & $28(45.2 \%)$ & $26(57.8 \%)$ & $11(23.4 \%)$ & $34(54.8 \%)$ & $99(45.8 \%)$ \\
\hline empirica & $27(43.5 \%)$ & $19(42.2 \%)$ & $32(68.1 \%)$ & $21(33.9 \%)$ & $99(45.8 \%)$ \\
\hline dopo antibiogramma & $7(11.3 \%)$ & 0 & $4(8.5 \%)$ & $7(11.3 \%)$ & $18(8.3 \%)$ \\
\hline $\begin{array}{l}\text { Adesione linee guida regionali di } \\
\text { profilassi antibiotica in chirurgia }\end{array}$ & $5(17.9 \%)$ & $7(26.9 \%)$ & $2(18.2 \%)$ & $20(58.8 \%)$ & $34(34.3 \%)$ \\
\hline
\end{tabular}


Tabella 3. Infezioni nosocomiali

\begin{tabular}{|c|c|c|c|c|c|c|c|c|c|}
\hline 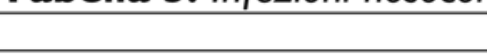 & \multicolumn{2}{|c|}{2007} & \multicolumn{2}{|c|}{2008} & \multicolumn{2}{|c|}{2009} & \multicolumn{2}{|c|}{2010} & TOTALE (\%) \\
\hline Infezioni riscontrate/anno & \multicolumn{2}{|c|}{$19(22.9 \%)$} & \multicolumn{2}{|c|}{$43(47.3 \%)$} & \multicolumn{2}{|c|}{$31(33.3 \%)$} & \multicolumn{2}{|c|}{$21(26.9 \%)$} & $114(33.0)$ \\
\hline Colture effettuate/anno & \multicolumn{2}{|c|}{$16(84.2 \%)$} & \multicolumn{2}{|c|}{$4(9.3 \%)$} & \multicolumn{2}{|c|}{$9(29.0 \%)$} & \multicolumn{2}{|c|}{$9(42.9 \%)$} & $38(33.3)$ \\
\hline INFEZIONI NOSOCOMIALI & \multicolumn{2}{|c|}{$8(9.6 \%)$} & \multicolumn{2}{|c|}{$7(7.7 \%)$} & \multicolumn{2}{|c|}{$10(10.8 \%)$} & \multicolumn{2}{|c|}{$6(7.7 \%)$} & $31(9.0)$ \\
\hline Terapia intensiva & \multicolumn{2}{|c|}{$5(62.5 \%)$} & \multicolumn{2}{|c|}{0} & \multicolumn{2}{|c|}{$2(20.0 \%)$} & \multicolumn{2}{|c|}{$4(66.7 \%)$} & II (35.5) \\
\hline Area medica & \multicolumn{2}{|c|}{$2(25.0 \%)$} & \multicolumn{2}{|c|}{$2(28.6 \%)$} & \multicolumn{2}{|c|}{$5(50.0 \%)$} & \multicolumn{2}{|c|}{$2(33.3 \%)$} & II (35.5) \\
\hline Area chirurgica & \multicolumn{2}{|c|}{ I (12.5\%) } & & $1.4 \%)$ & & $0.0 \%)$ & & 0 & $9(29.0)$ \\
\hline Infezioni basse vie respiratorie & 4 & & 4 & & 2 & & 2 & & $12(38.7)$ \\
\hline Colture effettuate & 4 & & 0 & & 0 & & 2 & & 6 \\
\hline Colture positive & 3 & & 0 & & 0 & & 2 & & 5 \\
\hline Microrganismi isolati: & & & & & & & & & \\
\hline A. baumannii & 1 & & & & & & & & 1 \\
\hline P. aeruginosa & 1 & & & & & & 1 & & 2 \\
\hline P. caepacea & $\mathrm{I}$ & & & & & & & & $\mathrm{I}$ \\
\hline P. mirabilis & & & & & & & 1 & & 1 \\
\hline Batteriemie & 2 & & & & 5 & & 3 & & $10(32.3)$ \\
\hline Colture effettuate & 2 & & & & 2 & & 3 & & 7 \\
\hline Colture positive & 2 & & & & 2 & & 3 & & 7 \\
\hline Microrganismi isolati: & & & & & & & & & \\
\hline E. coli & 1 & & & & & & & & 1 \\
\hline K. pneumoniae & 1 & & & & I & & 1 & & 3 \\
\hline S. epidermidis & & & & & $\mathrm{I}$ & & & & $\mathrm{I}$ \\
\hline A. baumannii & & & & & & & 1 & & 1 \\
\hline MRSA & & & & & & & 1 & & 1 \\
\hline Infezioni cute/connettivo & I & & 3 & & & & & & $4(12.9)$ \\
\hline Colture effettuate & 1 & & 0 & & & & & & 1 \\
\hline Colture positive & $\mathrm{I}$ & & 0 & & & & & & $\mathrm{I}$ \\
\hline Microrganismi isolati: & & & & & & & & & \\
\hline S. aureus & 1 & & & & & & & & 1 \\
\hline Infezioni della vie urinarie & I & & & & & & I & & $2(6.5)$ \\
\hline Colture effettuate & 1 & & & & & & 1 & & 2 \\
\hline Colture positive & $\mathrm{I}$ & & & & & & $\mathrm{I}$ & & 2 \\
\hline Microrganismi isolati: & & & & & & & & & \\
\hline K. pneumoniae & 1 & & & & & & & & I \\
\hline E. faecalis & & & & & & & 1 & & 1 \\
\hline Infezioni del tessuto osseo & & & & & $\mathbf{I}$ & & & & I (3.2) \\
\hline Colture effettuate & & & & & 0 & & & & 0 \\
\hline Sede non determinata & & & & & 2 & & & & $2(6.5)$ \\
\hline Colture effettuate & & & & & 0 & & & & 0 \\
\hline
\end{tabular}

\section{BIBLIOGRAFIA}

1. Linee guida per la profilassi antibiotica in chirurgia Delibera A.Re.S. n. 83 del 30/07/04.

2. Lizioli A, Privitera G, Alliata E et al. Prevalence of nosocomial infections in Italy: result from the Lombardy survey in 2000. J Hosp Infect. 2003; 54: 141-8.

3. Pavia M, Bianco A, Viggiani NM, Angelillo IF. Prevalence of hospital-acquired infections in Italy. $J$ Hosp Infect. 2000; 44: 135-9.
4. Pellizzer G, Mantoan P, Timillero L, et al. Prevalence and risk factors for nosocomial infections in hospitals of the Veneto region, north-eastern Italy. Infection. 2008; 36: 112-9.

5. Villone G, Salvante D. Sorveglianza e controllo delle infezioni ospedaliere in Puglia. Progetto Sanità 2007; 19: 2-64

6. Zotti CM, Messori Ioli G, Charrier L, et al. Hospitalacquired infections in Italy: a region wide prevalence study. J Hosp Infect. 2004; 56: 142-9. 